\title{
Digital in FFL (French as a foreign language) class: how to make our learners think?
}

\section{[Le numérique en classe de FLE: comment agir pour amener nos apprenants à réfléchir ?]}

\author{
Olga M. Kozarenko
}

DOI: 10.18355/XL.2018.11.01XL.20

\begin{abstract}
The new technologies are more and more invading the educational area including the area of modern languages teaching-learning. It spreads is so fast that sometimes we are not able to adapt to them.

What are the challenges of students and the problems of the teachers, who sometimes claim of a strong dependence on digital tools of their students?

The research in question represents the results of the work carried out with teachers of the FFL as well as their Russian learners with linguistic and non-linguistic preparation.

The use of qualitative and quantitative methods has allowed us to understand better the weak points of the teachers of French to teach the FL (foreign language) profile. Surveys of our students have completed the image of the new educational landscape.

A thorough data analysis helped us to find tracks of reflections and avoid errors in the practical use of new technologies in the course of FL.
\end{abstract}

Key words: language training, new technologies, digital tools, advantage and disadvantages of digital

\section{Résumé}

Les nouvelles technologies envahissent de plus en plus l'espace éducatif y compris le domaine de l'enseignement-apprentissage des langues vivantes. Elles se propagent à une telle vitesse que parfois nous n'arrivons pas à nous y adapter.

Quelles sont les difficultés des étudiants et les préoccupations des enseignants qui constatent parfois une forte dépendance aux outils numériques de leurs apprenants ?

$\mathrm{La}$ recherche en question présente les résultats du travail réalisé auprès des enseignants du FLE ainsi que de leurs apprenants universitaires russophones de formation linguistique et non-linguistique.

L'usage des méthodes qualitatives et quantitatives nous a permis de mieux comprendre les points faibles des enseignants de français pour enseigner les LV médiatisées. Les sondages des étudiants ont complété l'image du nouveau paysage éducatif.

L'analyse approfondie des données nous a aidé à élaborer des traces de réflexions et à trouver les erreurs à éviter dans l'usage des nouvelles technologies aux cours de langues étrangères.

Mots-clés : formation linguistique, nouvelles technologies, outils numériques, avantages et inconvénients du numérique

\section{Introduction}

\section{I.1 Contexte général de la recherche}

En Fédération de Russie les Langues Vivantes sont enseignées obligatoirement au Bakalavriat (Licence) et en Magistrature (Master) indépendamment de la formation. C'est bien entendu la langue anglaise qui se positionne comme première langue étrangère dans tous les établissements. En même temps le français reste la première 
langue vivante dans les établissements de formation linguistique ou internationale. C'est la formation non-linguistique qui est majoritaire chez les apprenants du FLE. Malheureusement le nombre d'étudiants qui choisissent le français reste parmi les plus modestes par rapport aux autres langue étrangères.

\section{I.2 Difficultés d'enseignement}

Même si les professeurs de français sont très enthousiastes pour transmettre la langue française et ouverts à toutes les technologies innovantes il y a des facteurs qui compliquent leur travail. Certains d'entre eux sont présentés ci-dessous.

De multiples réformes de l'éducation ces dernières années détournent les enseignants de la nécessité de s'y adapter.

On constate la réduction permanente de la quantité de cours de langues vivantes. On les étudie normalement 3 ou 4 semestres dans les établissements de formation non-linguistique avec 3 heures académiques par semaine.

La charge horaire des enseignants est perpétuellement en hausse ce qui diminue l'attractivité de ce métier pour les jeunes spécialistes et accélère la fatigue professionnelle.

L'universalité de la langue anglaise réduit l'attrait de l'apprentissage des secondes langues étrangères, pas tant chez les étudiants que surtout chez les fonctionnaires dans le domaine de l'éducation.

\section{3. TICE dans enseignement-apprentissage des $L V$ dans la recherche des auteurs russophones et étrangers}

Le sujet d'usage des TICE en éducation devient de plus en plus populaires parmi les chercheurs russophones. L'étude des recueils d'articles des colloques qui ont eu lieux à trois universités de Moscou consacrés à l'enseignement/apprentissage des LV (Kozarenko, 2016) a relevé les priorités langagières des chercheurs russophones (analyse de près de soixante-dix d'articles). Dans les articles consacrés à l'usage des TICE dans le domaine des LV, 86\% traitaient de l'expérience des professeurs d'anglais. Les articles des professeurs d'allemand, de français et d'espagnol ne représentaient respectivement que $7 \%, 5 \%$ et $2 \%$.

Les sujets dominants dans la recherche sur les TICE étaient : «Usage des ressourcesInternet et des dictionnaires en ligne » - un cinquième des articles étudiés ; «Pédagogie technologique »- la sixième partie des articles. Ces sujets étaient suivis (moins de 10\%) par " Philosophie de l'enseignement linguistique »; «Enseignement intégré »; "Communication interculturelle à l'époque numérique »; "Potentiel didactique de WEB 2,0»; "TIC dans la recherche linguistique »; "SMART technologies »; « Enseignement/apprentissage des LV à la distance ».

Parmi les ouvrages des auteurs russophones dans le domaine d'usage de nouvelles technologues dans l'enseignement-apprentissage des langues étrangères on peut nommer les livres de Titova S. V. (Titova, 2017 ; 2014 ; Titova, Filatova, 2014 ) qui résultent l'expérience de plusieurs années de recherche dans le domaine de l'utilisation des technologies informatiques dans l'enseignement des langues étrangères à l'université et où on peut trouver des conseils sur la création de sites web éducatifs, sur l'évaluation des ressources en ligne, ainsi que de leur usage efficace dans le processus de formation. Nazarenko A.L. (2014) a systématisé les principes d'éducation et de formation des spécialistes à la distance. Les ouvrages de Kashchuk (2014 a, b) visent les particularités de l'intégration des technologies Web 2.0 dans le processus d'apprentissage de FLE. Un nouveau modèle de la compétence de recherche à l'époque de WEB 2,0 et son application pour les langues étrangères a été élaboré par Karsanti, T. et Kozarenko, O. (2016).

Le numérique facilite l'accès aux multiples ressources en ligne, propose des plateformes pour le dépôt des documents virtuels, et favorise à l'époque du WEB 2,0 les contacts entre les apprenants et les enseignants joue un rôle tout à fait particulier 
dans l'enseignement-apprentissage des LV. N. Guichon (Guichon, 2012) a élaboré une méthode d'apprentissage des langues médiatisés par les technologies. On porte une attention particulière au numérique pour l'enseignement et l'apprentissage des langues pour non-spécialistes (Demaiziere - Grisbois, 2014 ; Grosbois, M. 2015 ; Demaizière F. - Grosbois, M. 2014). Les chercheurs constatent que l'usage de nouvelles technologue rend plus complexe l'accompagnement de l'apprentissage des LV (Ciekanski, 2014).

La majorité des articles sur ce sujet montrent l'utilité de nouvelles technologies à travers l'intensification, l'individualisation, et la systématisation du travail des apprenants. En même temps les auteurs attirent attention aux problèmes d'usage des technologies numériques. Par exemple SHadrikov, SHemet (2009) constatent que l'usage massif des technologies en éducation (qui est inévitable) demande une attention particulière par rapport à ses conséquences négatives qui doivent être étudiées. Les particularités de la psychologie de l'Internet sont traitées par Vojskunskij, A. E. (2005) qui synthétise les recherches des auteurs russophones qui ont étudié les conséquences positives et négatives (la dépendance pathologique de l'Internet, la dissinkhronie du développement etc.). Certains problèmes d'efficacité de la formation à la distance sont traités dans l'ouvrage de Borisova (2016).

\section{I.4. Problématique de la recherche}

Sans nier tous les avantages des technologies numériques on a noté simultanément les préoccupations des enseignants des LV liées à la manière d'utiliser des outils numériques par leurs apprenants.

Ainsi, les facteurs qui nous ont incité à effectuer cette recherche étaient les suivants. Globalement, la compréhension de la nécessité du changement de paradigme d'enseignement des LV avec l'usage massif de nouvelles technologies ainsi que l'envie de mieux comprendre comment profiter des enjeux de la pédagogie numérique. Plus spécifiquement - c'est le constat d'une forte dépendance de certains apprenants aux appareils numériques, ce qui parfois est même considérée comme une perte partielle de leur autonomie intellectuelle.

\section{Hypothèse de la recherche}

La recherche en question nous permet de mieux comprendre l'utilisation des outils numériques par les apprenants en cours de FLE et leur manière de réfléchir avec les appareils numériques.

L'étude de l'opinion des professeurs et la compréhension de leur trajectoire individuelle dans la pédagogie numérique permettra de mieux comprendre la nature de leurs préoccupations, ce qui aidera à rétablir l'équilibre du processus éducatif.

\section{Méthodes de la recherche}

Pour la réalisation de nos études on a utilisé les méthodes qualitatives ainsi que quantitatives. Pour la collecte des données on s'est référé à l'enquête en ligne. Le questionnaire a été rédigé pour les professeurs de FLE des institutions de l'enseignement supérieur. Il s'adressait aux professeurs des Universités pédagogiques et linguistiques qui formaient des enseignants de français et des linguistes ainsi qu'aux professeurs universitaires formant des non-spécialistes, soit 50 interrogés.

Pour compléter les données statistiques et les personnaliser nous avons effectué des entretiens individuels.

La même méthode mixte a été utilisée pour les apprenants : 70 interrogés.

Les données chiffrées étaient systématisées permettant de réaliser une analyse descriptive et comparative présentée sous forme des tableaux et des graphiques ce qui nous a aidé à éclaircir la problématique initiale.

\section{Résultats du sondage} IV.1. Apprenants. 


\section{IV.1.1. Apprenants. Contexte général.}

Pour mieux comprendre les résultats de la recherche observons les caractéristiques des personnes interrogées. $47 \%$ d'entre elles sont débutants en français suivis par les avancés $(30 \%)$, le niveau intermédiaire ne représentant que $23 \%$. Leur motivation pour étudier une deuxième langue étrangère était haute pour la moitié des interrogés, pour $41 \%$ elle était moyenne et basse pour $9 \%$.

Les réponses à la question «La seconde LV (SLV) me pose plus de difficultés, car ...:» sont présentées dans le Graphique 1. Ils montrent que les étudiants sont assez critiques par rapports à eux-mêmes, mais que cependant ils aimeraient avoir plus de cours. $20 \%$ trouvent l'étude de la seconde LV difficile.

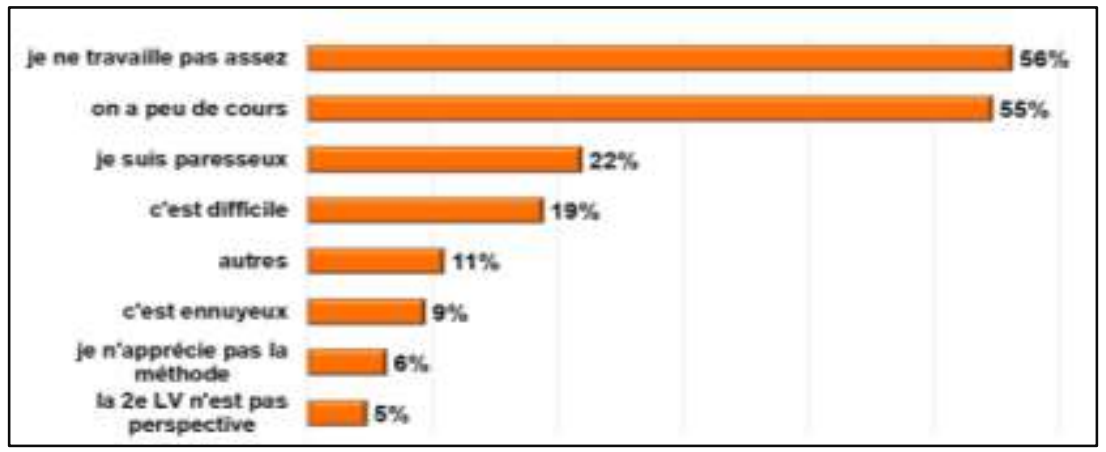

\section{Graphique 1 : Les raisons des difficultés des étudiants pour apprendre une seconde langue étrangère}

IV.1.2. Résultats de l'enquête sur l'usage des outils numériques par les étudiants en cours de $L V$.

Les étudiants recourent à leurs smartphones parce qu'ils considèrent que cela rend leur travail plus efficace (62\%) et d'un point de vue psychologique ils se sentent plus sûrs d'eux. Néanmoins $6 \%$ disent que l'usage des appareils numériques leur empêche à se concentrer.

A la question concernant ce qu'ils éprouvent en cours de LV2 sans appareil électronique (AE), la moitié des interrogés constate un inconfort $(37 \%)$ et même un grand inconfort (12\%) (Tableau 1).

\begin{tabular}{|c|c|}
\hline Réponses des étudiants & $\%$ \\
\hline Grand inconfort & 12 \\
\hline Je ne suis pas à l'aise & 37 \\
\hline Ça m'est égal & 22 \\
\hline Je me débrouille tranquillement & 27 \\
\hline Autre & 2 \\
\hline
\end{tabular}

XLinguae, Volume 11 Issue 1XL, January 2018, ISSN 1337-8384, eISSN 2453-711X 
Tableau 1: Les sentiments, éprouvés par les étudiants en cours de LV2 quand ils travaillent sans leurs appareils électroniques

Durant le travail les professeurs de FLE ont remarqué que les travaux écrits des étudiants faits à la maison présentaient souvent soit la traduction en français des textes russes réalisés avec les traducteurs en ligne (https://translate.yandex.ru) soit les copier-coller des textes authentiques. C'est pourquoi il nous est apparu intéressant de comprendre l'algorithme du travail des étudiants dans ce cas. On l'a découvert avec leurs réponses présentées dans le Graphique 2.

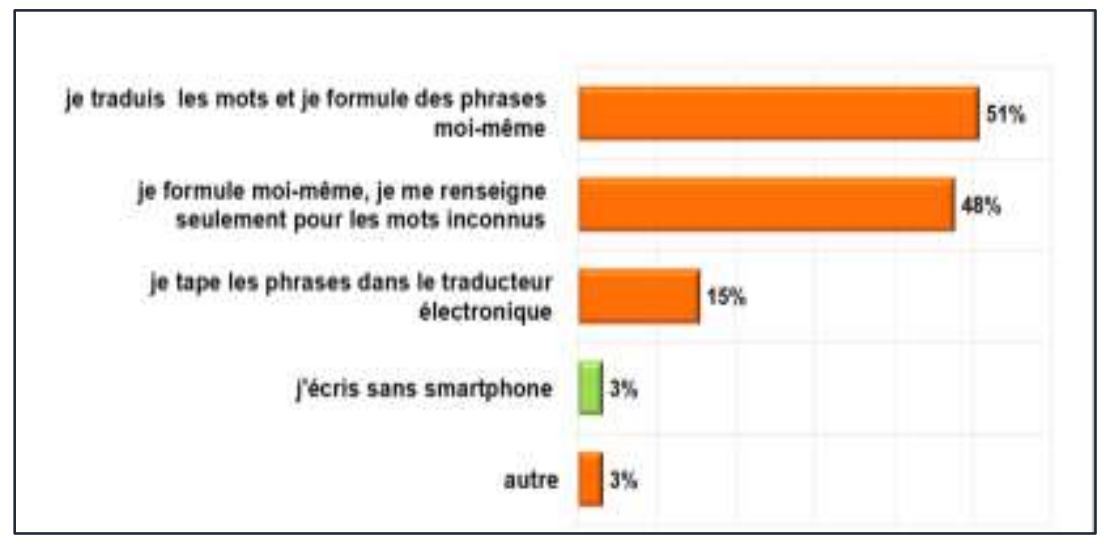

\section{Graphique 2: L'algorithme du travail autonome des étudiants pour la rédaction des expressions écrites}

La majorité des étudiants d'après leurs réponses recourent aux $\mathrm{AE}$ pour consulter le dictionnaire en ligne pour rechercher des mots inconnus soit avant la formulation des phrases des textes, soit lors de ce travail. $15 \%$ tapent les phrases dans le traducteur en minimisant leurs efforts d'apprentissage. Ceux qui n'utilisent pas le smartphone pour la production écrite sont en minorité (3\%).

Quel type de phrase est préférable pour les étudiants ? 55\% comprennent que cela dépend du style de communication. En même temps $26 \%$ utilisent les phrase courtes et simples et seulement $13 \%$ formulent les phrases longues et complexe.

A la question «En cours de LV, faut-il apprendre à écrire tout de suite à l'ordinateur?» $68 \%$ ont donné une réponse négative. L'ordinateur a été choisi seulement par $7 \%$ des personnes. $22 \%$ des interviewés ne voyaient pas de grande différence.

Les professeurs constatent que pendant les réponses orales des étudiants en classe de FLE avant de réponde les étudiants cherchent la possibilité de consulter leurs AE. C'est pourquoi nous avons formulé la question suivante : "Quelle est votre première réaction quand vous devez répondre oralement en LV (répondre à la question, s'exprimer etc.) ? 73\% disent qu'ils se concentrent et réfléchissent sans smartphone, $25 \%$ s'avouent sincèrement que leur main se dirige vers leur smartphone.

Pourquoi les apprenants préfèrent-ils obtenir de l'aide de l'appareil électronique lors de leurs réponses orales? (Tableau 2) : 


\begin{tabular}{|c|c|}
\hline Réponses des étudiants & $\%$ \\
\hline Je ne suis pas sûr de moi & $51 \%$ \\
\hline C'est plus vite & $29 \%$ \\
\hline «Il» sait mieux le & $29 \%$ \\
\hline Je ne veux pas réfléchir & $11 \%$ \\
\hline C'est mon habitude & $14 \%$ \\
\hline Autre & $15 \%$ \\
\hline
\end{tabular}

Tableau 2: Les raisons d'usage des appareils électroniques lors de la réponse orale des étudiants

Les motives d'usage des outils numériques dans ce cas varient en fonction des caractéristiques personnelles - l'incertitude (la moitié des réponses) et « je ne veux pas réfléchir » (11\%) ainsi que du style du travail. Les étudiants considèrent que l'AE économise leur temps et ils croient à la fiabilité de l'information virtuelle plus qu'à leur capacité d'interagir. Dans la formulation «Autre» les réponses étaient les suivantes : «Je préfère obtenir l'aide d'un appareil électronique lors de ma réponse pour : «Trouver un mot inconnu (ou oublié) dans le dictionnaire (électronique)»; «Je ne préfère pas, j'aime bien faire travailler mon cerveau»; "J'utilise mon appareil électronique uniquement quand je fais mes devoirs »; «Je pose la question à mon professeur »; « Je ne sais pas comment traduire »; «Moi, je ne préfère pas ça ». Une autre grande difficulté constatée par les professeurs de FLE était celle que les étudiants n'arrivaient pas à mémoriser correctement le nouveau lexique. Nous avons essayé de comprendre les raisons de ce phénomène. On a questionné les étudiants afin de savoir pourquoi ils avaient du mal à mémoriser les mots étrangers. Leurs réponses sont présentées sur le Graphique 3. 


\section{J'ai du mal à mémoriser les mots étrangers parce que:}

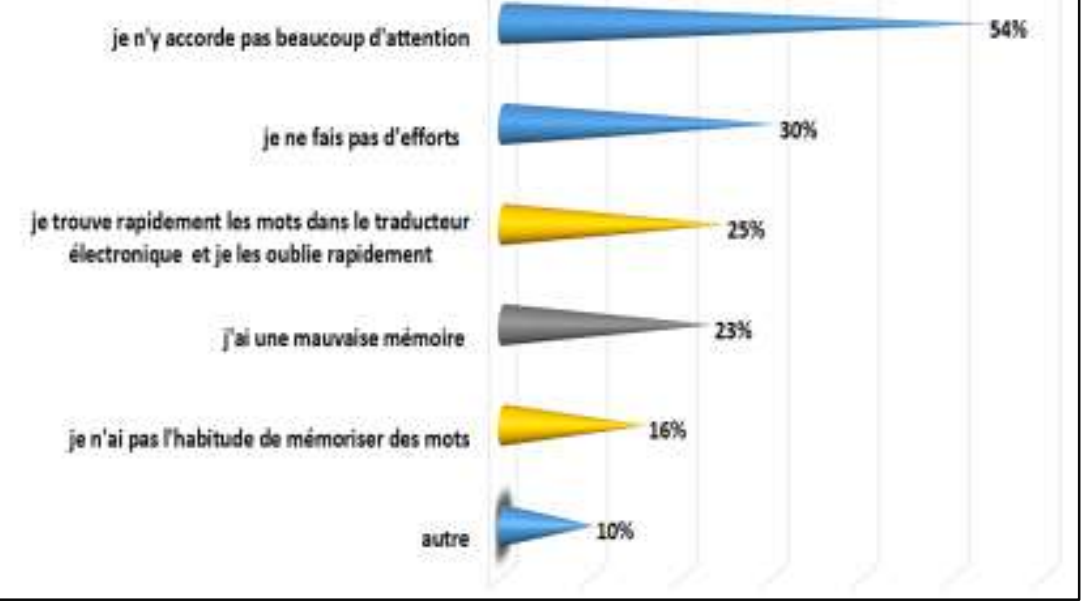

Graphique 3: Les explications des étudiants sur pourquoi ils mémorisent mal les mots étrangers

Il est évidant qu'ayant un smartphone sous la main les étudiants n'accordent pas beaucoup d'attention à la mémorisation des mots (54\%) et même n'ont pas l'habitude de le faire $(16 \%)$. Un quart des étudiant a avoué trouver rapidement les mots dans les dictionnaires ou traducteurs électroniques, mais qu'ils les oubliaient aussi vite. Presque la même quantité d'étudiants a constaté avoir une mauvaise mémoire. Seulement $10 \%$ des interrogés ont écrit dans l'option « Autre » qu'ils n'avaient pas de difficultés à mémoriser les mots étrangers.

Est-ce que les étudiants utilisent les applications numériques pour mémoriser le nouveau lexique ? Le Graphique 4 illustrent leurs réponses qui montrent que $60 \%$ des apprenants ne le font pas.

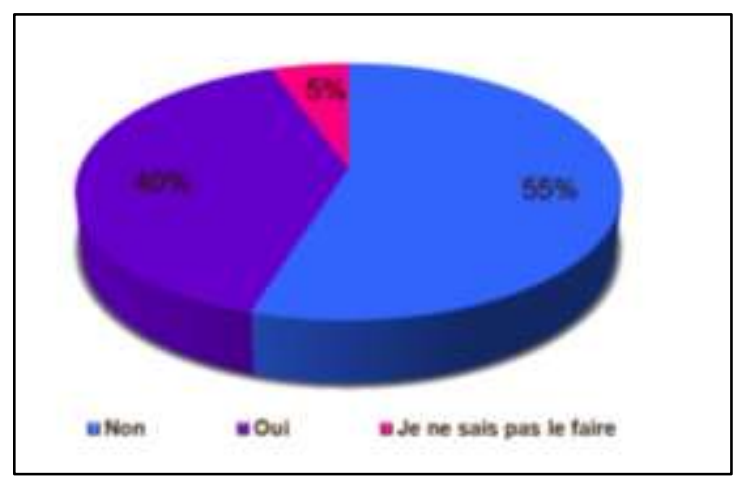

Graphique 4: Usage des applications numériques pour mémoriser le lexique

Mais est-ce que les professeurs prévoient le travail avec les applications pendant les cours de FLE ? (Graphique 5). Les statistiques montrent que pour seulement $7 \%$ des 
professeurs c'est une condition obligatoire. 57\% des enseignants le font rarement, voire pas du tout.

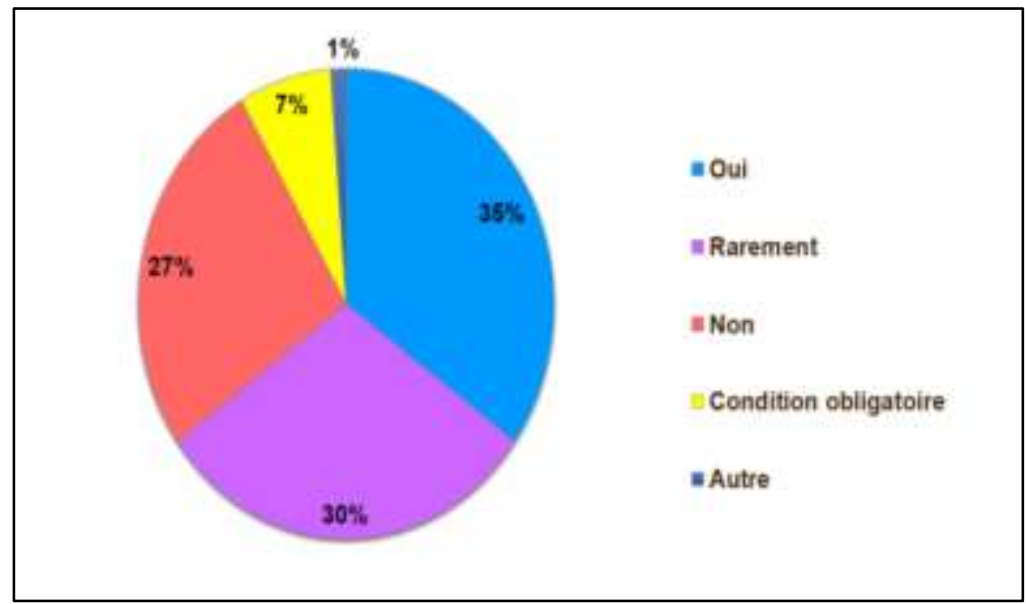

Graphique 5: Usage des applications numériques pour faciliter la mémorisation du lexique en cours de FLE

Les étudiants, s'adressent-ils souvent aux enseignants pour trouver telle ou telle application? (Tableau 3). D'après leurs réponses les apprenants sont très autonomes dans ce domaine. Deux fois plus souvent ils trouvent les applications eux-mêmes (Tableau 3).

\begin{tabular}{|c|c|}
\hline Réponses des étudiants & \% \\
\hline Je les trouve moi-même & 78 \\
\hline Le professeur de LV & 42 \\
\hline Les autres & 26 \\
\hline
\end{tabular}

Tableau 3: Les réponse des étudiants à la question « Vous utilisez les applications et les sites recommandés par : ..., »\%

Quels sentiment les étudiants éprouvent-ils si le professeur ne permet pas d'utiliser un appareil électronique lors de leurs réponses? (Graphique 6). 


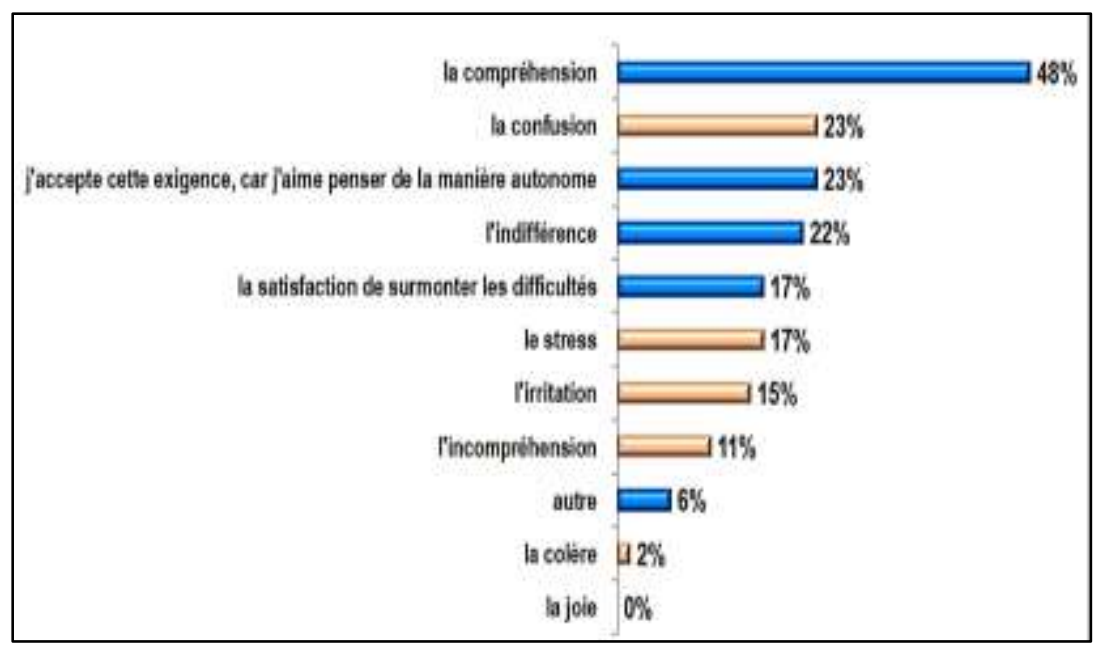

Graphique 6: Les sentiments des étudiants à l'interdiction d'usage de l'appareil électronique lors de la réponse.

Près de la moitié des interrogés acceptait les exigences des professeurs (48\%) et même comprenait que cela était important pour leur développement (23\%). $15 \%$ des étudiants éprouvaient la satisfaction de réfléchir. 22\% restaient indifférents. Avec une autre couleur sur le graphique nous avons essayé de montrer les sentiments négatifs des apprenants qui en somme ont atteint un chiffre assez important (68\%). C'était la confusion, l'incompréhension, l'irritation et même le stress et la colère (2 $\%)$.

Comment les étudiant apprécient-ils eux-mêmes le développement de leur capacités cognitives lors des cours des LV? (Graphique 7). Nous avons proposé aux étudiants de procéder une auto-évaluations d'après des critères prédéfinis et accessibles.

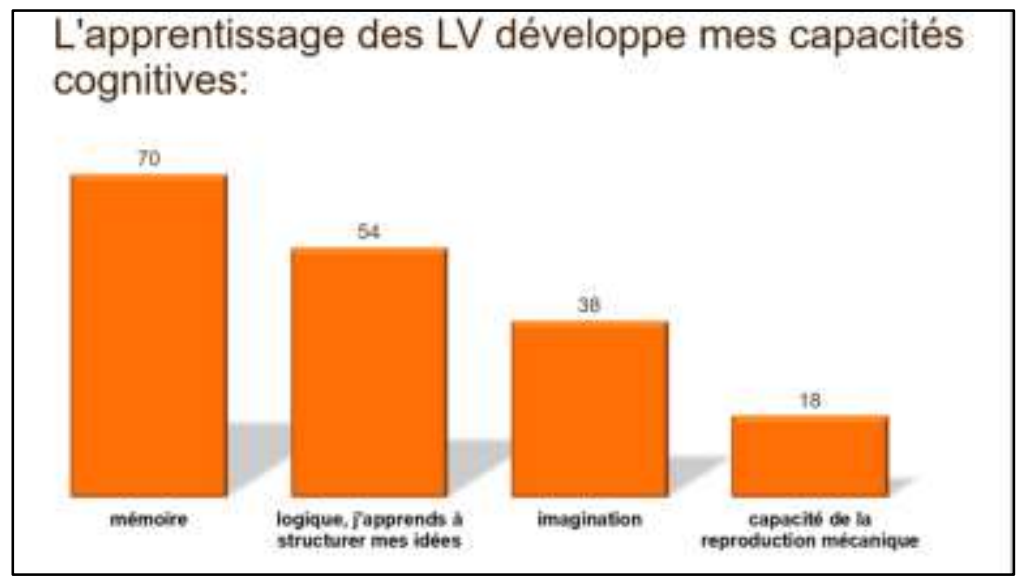

Graphique 7: Les opinions des étudiants sur le développement de leurs capacités cognitives pendant l'apprentissage des $\mathrm{LV}$. 
Les résultats statistiques illustrent un pourcentage assez élevé du développement de la mémoire $(70 \%)$ et de la logique $(50 \%)$. La compétence de bas niveau (reproduction mécanique) représente $18 \%$ et reflète en partie les particularités de l'apprentissage des langues étrangères.

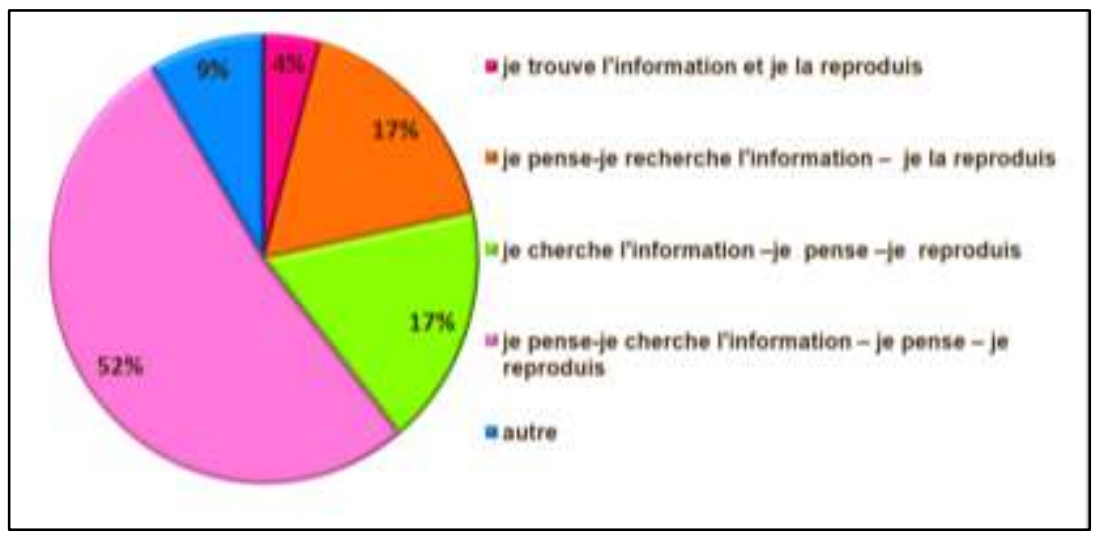

\section{Graphique 8: L'algorithme de la reproduction de l'information en LV par les étudiants}

Pour mieux comprendre le style du travail des étudiants lors de leur apprentissage des $\mathrm{LV}$, les étapes où ils interviennent pour modifier/personnaliser l'algorithme de leur travail par rapport à leurs demandes/besoins personnels nous avons proposé plusieurs pistes à étudier. Les variantes des réponses représentaient l'augmentation de la nécessité de comprendre que chaque étape du travail doit être repensée par l'apprenant et servir ses propres intérêts et son développement cognitif.

Les réponses montrent que la reproduction mécanique en LV n'atteint que 4\%. La moitié des interrogés réfléchit/adapte chaque fois ses actions à ses besoins. Pour $34 \%$ une des étapes est effectuée mécaniquement: soit ils surfent sur la Toile à la recherche de l'informations et la modifient avant de la reproduire ; soit ils sont plus attentifs à la recherche de l'information qu'ils reproduisent ensuite sans la modifier. Mais il faut rester critique par rapport aux réponses des étudiants qui parfois aiment choisir des réponses correctes et ils l'avouent eux-mêmes dans les commentaires « Autre »: «La majorité va choisir la dernière option ». Il est à noter que les réponses à cette question ont provoqué des difficultés aux apprenants parce qu'ils font ce travail inconsciemment. Les étudiants sont-ils satisfaits de l'efficacité du développement de leurs capacités cognitives en cours de LV? La Graphique 9 représente leurs réponses sur le niveau de développement de leurs capacités cognitives en cours de LV.

Un tiers d'étudiants (la majorité des réponses) pensent que seulement $25 \%$ de leur potentiel intellectuel est sollicité en cours de LV. 15\% des interrogés ont le sentiment qu'ils développent au maximum leurs capacités intellectuelles. Le chiffre de ceux qui constatent un faible développement $(10 \%)$ de leurs compétences cognitives en cours de FLE est $23 \%$. 


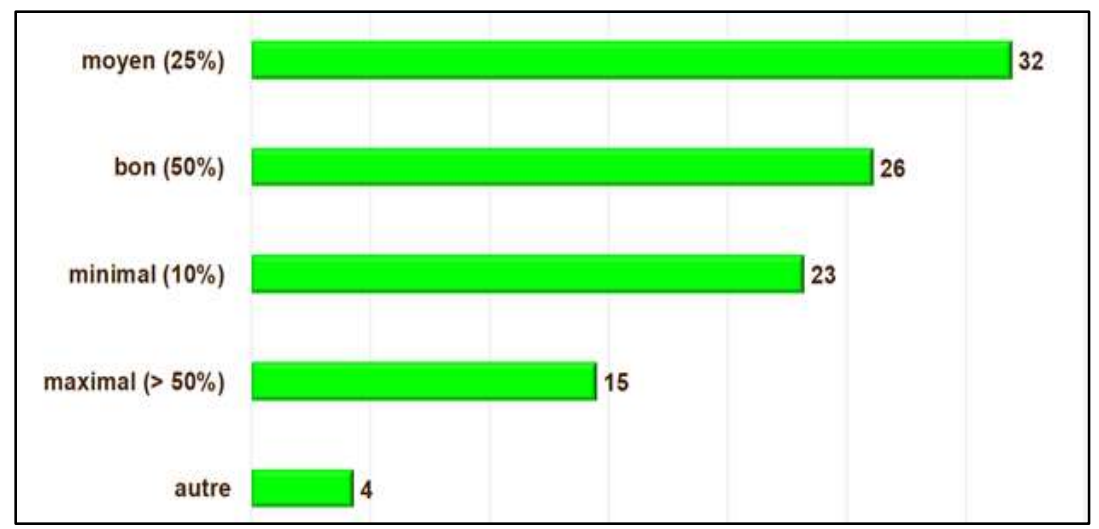

Graphique 9: La perception personnelle des étudiants sur le niveau de développement de leurs capacités cognitives en cours de LV

Pour les étudiants la forme la plus efficace d'apprentissage des LV est le cours individuel avec un professeur. Graphique 10. Les cours en ligne et le travail autonome n'atteignent qu'un pour cent.

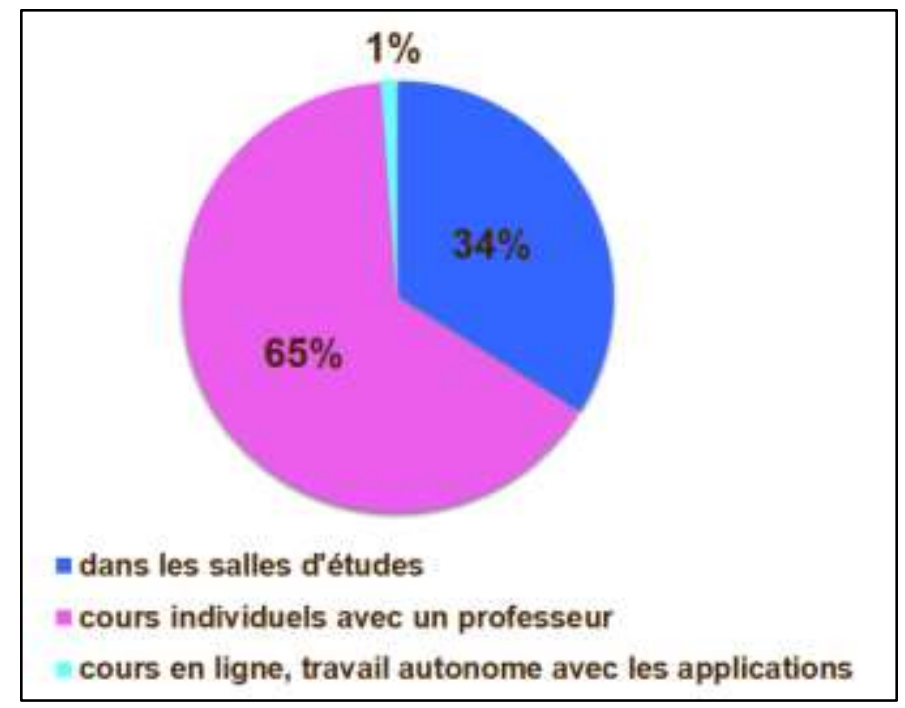

Graphique 10: Les opinions des étudiants sur la forme la plus efficace d'apprentissage des LV.

Les apprenants préfèrent les consultations avec le professeur dans les salles d'études (80\%) par rapport aux moyens numériques. Graphique 11. 


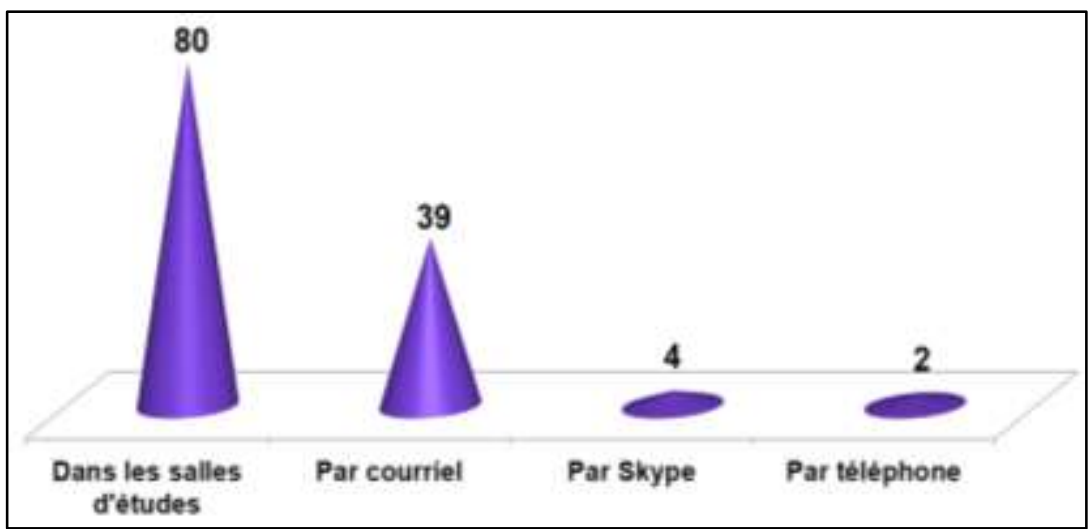

\section{Graphique 11: Les formes des consultations préférées par les étudiants.}

Compte tenu du développement massif des nouvelles technologies, des traducteurs en ligne de plus en plus efficace comment les étudiants voient-ils l'avenir de l'enseignement/apprentissage des LV ? Graphique 12.

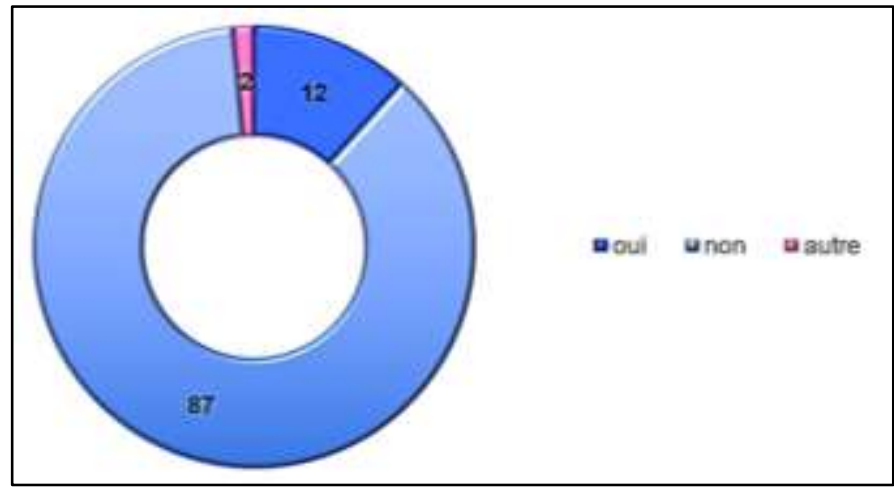

Graphique 12: «À l'avenir, avec le développement des technologies on n'aura pas besoin d'apprendre les $\mathrm{LV}, \%$ »

D'après leurs réponses la révolution numérique ne remplacera pas la nécessité d'apprendre les langues étrangères (87\%).

\section{IV.1.3. Apprenants. Discussion.}

L'analyse des résultats du sondage des étudiants montrent qu'ils :

comprennent l'efficacité des outils numérique lors de l'apprentissage des LV ;

se sentent plus sûrs avec des appareils électroniques ;

ne se voient pas dépendants des appareils électroniques ;

sont persuadés qu'ils s'adressent aux appareils électroniques en cas de nécessité ;

les préfèrent du fait de leur rapidité et de l'accès à l'information ;

se voient plus compétents pour la recherche informatique que les professeurs ; 
comprennent que l'apprentissage de LV développe leurs compétences cognitives ;

En même temps :

La majorité d'entre eux (68\%) éprouvent des sentiments négatifs s'ils ne peuvent pas utiliser leurs smartphones lors des réponses.

On constate un haut pourcentage d'incertitude des étudiants en eux-mêmes, s'ils travaillent sans $\mathrm{AE}$ avec le sentiment qu'un gadget « sait mieux » (80 $\%)$.

Avec les $\mathrm{AE}$ les étudiants se libèrent du travail routinier (la mémorisation des mots), en perdant en même temps l'entrainement de leur mémoire ( $23 \%$ constatent qu'ils ont une mauvaise mémoire). L'information trouvée rapidement s'oublie aussi rapidement sans entrainements supplémentaires.

Les étudiants ne sont pas toujours efficaces pour choisir le meilleur algorithme de leur activité éducative avec les outils numériques : quand il s'agit de la recherche de l'information en LV sur Internet ou le traitement de l'information trouvée.

Le minimum reste autonome en réalisant la production écrite en LV à domicile.

Les étudiants préfèrent l'assistance humaine et pas virtuelle dans leur accompagnement pédagogique (travail en présentiel, consultations dans les salles d'études).

$60 \%$ des interrogés suggèrent que le travail pour le développement de leurs compétences cognitives, d'après leur perception personnelle n'est pas très efficace en cours de LV (il leur manque l'interactivité et l'usage des applications)

Donc on voit la complexité du processus d'usage des TICE aux cours des LV non seulement au niveau des techniques éducatives, mais aussi au niveau psychologique et cognitif.

\section{IV.2. Professeurs.}

\section{IV.2. 1. Contexte général.}

Quel est le point de vue des professeurs sur le sujet en question ? Adressons-nous à la caractéristique générale des enseignants interrogés. Plus de $70 \%$ des professeurs avaient une grande ancienneté de travail soit plus de 20 ans ou entre 20 et 10 ans - (Graphique 13). Les jeunes enseignants ne représentaient que $5 \%$. Un quart d'étudiants a travaillé à l'université entre 5 et 10 ans. 


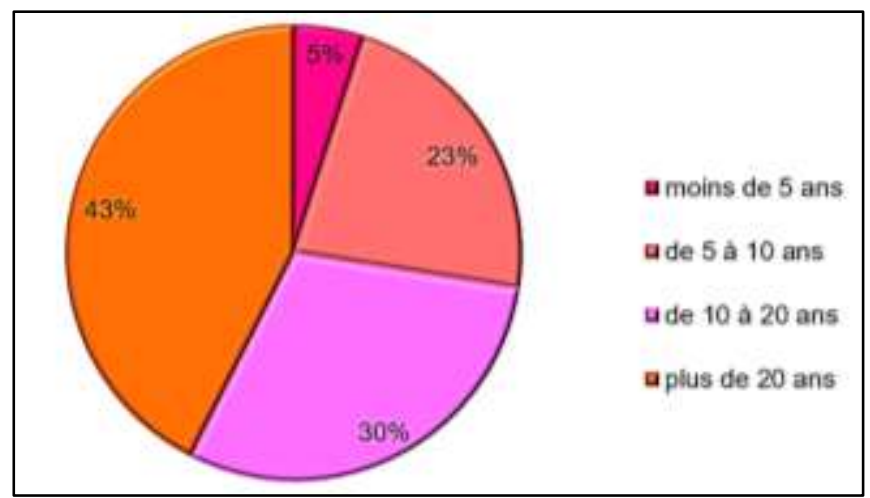

\section{Graphique 13: Ancienneté des enseignants interrogés.}

La moitié considérait leurs compétences informatiques comme bonnes $(50 \%)$ et même excellentes (23\%). Un quart des interrogés a choisi le niveau moyen et seulement $3 \%$ voyaient leurs compétences comme faibles.

$53 \%$ des professeurs utilisaient largement les documents numériques à leurs cours (à partir de 50\% par rapport à tous les documents). Pour $40 \%$ un tiers des documents était en forme numérique.

Quels facteurs empêchent les enseignants à utiliser les TICE lors de leurs cours ? (Graphique 14).

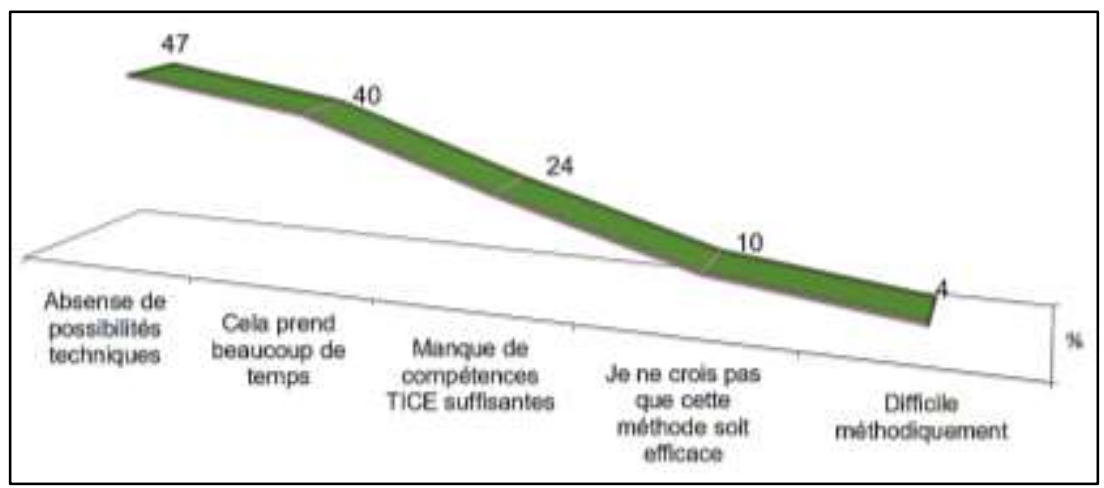

\section{Graphique 14 : Facteurs qui limitent l'usage des TICE par les enseignants en cours de LV}

Parmi les facteurs subjectifs c'est le facteur du manque de temps qui est au premier rang $(40 \%)$. On constate une contradiction entre la perception personnelle des compétences informatiques qui sont considérées comme bonnes et leur réalisation en pratique par les professeurs. Cela peut être lié à la modernisation permanente des outils numériques qui demande un perfectionnement constant. $10 \%$ ne croyaient pas à l'efficacité de ces méthodes.

\section{IV.2.2. Professeurs. Résultats du sondage.}

Lors des entretiens des enseignants ont formulé des problèmes relatifs à l'utilisation des TICE dont la liste est présentée ci-dessous : 
La commutation fréquente de l'attention du travail aux loisirs (jeu, messagerie, tchats).

Modification de l'algorithme de l'activité mentale : se renseigner devient plus important que réfléchir.

La facilité de « se renseigner » fait perdre l'habitude et la technique de « mémoriser».

On ne corrige plus le langage propre des étudiants mais celui transformé par les ON.

Dans le langage écrit on constate la simplification des énonciations langagières :

- Des phrases courtes et simples dominent dans les formulations des travaux écrits.

- L'Impératif remplace le Conditionnel.

- Un nivelage des registres langagiers.

- Une négligence dans l'écriture («l'ordinateur corrigera »).

Dans certains cas la traduction de la langue maternelle vers le français auprès des étudiants passe par l'anglais.

Les professeurs sont unanimes dans leurs réponses à la question, si les apprenants s'adressent plus souvent aux gadgets pour répondre (Graphique 15).

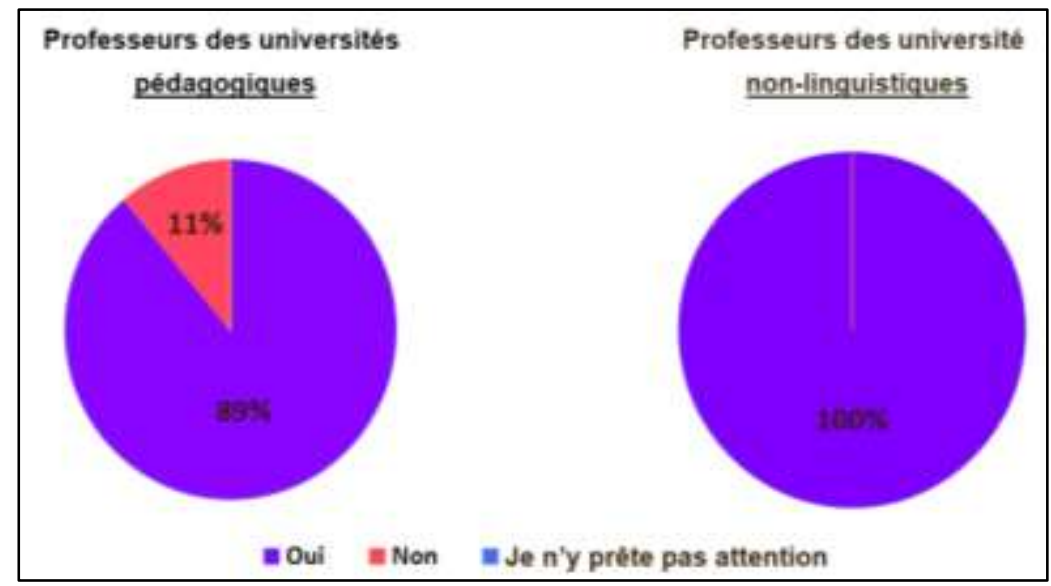

Graphique 15 : Réponses des enseignants à la suggestion : « Les étudiants, lors des réponses, recourent-ils plus souvent à un appareil électronique ?»

Dans quelles situations des professeurs permettent aux étudiants d'utiliser leurs appareils électroniques? (Graphique 16). Les statistiques démontrent que c'est la recherche d'informations qui reste l'activité principale pour le travail avec les outils numériques dans la salle d'études au cours de LV.

95\% des enseignants contrôlent l'utilisation des AE. 


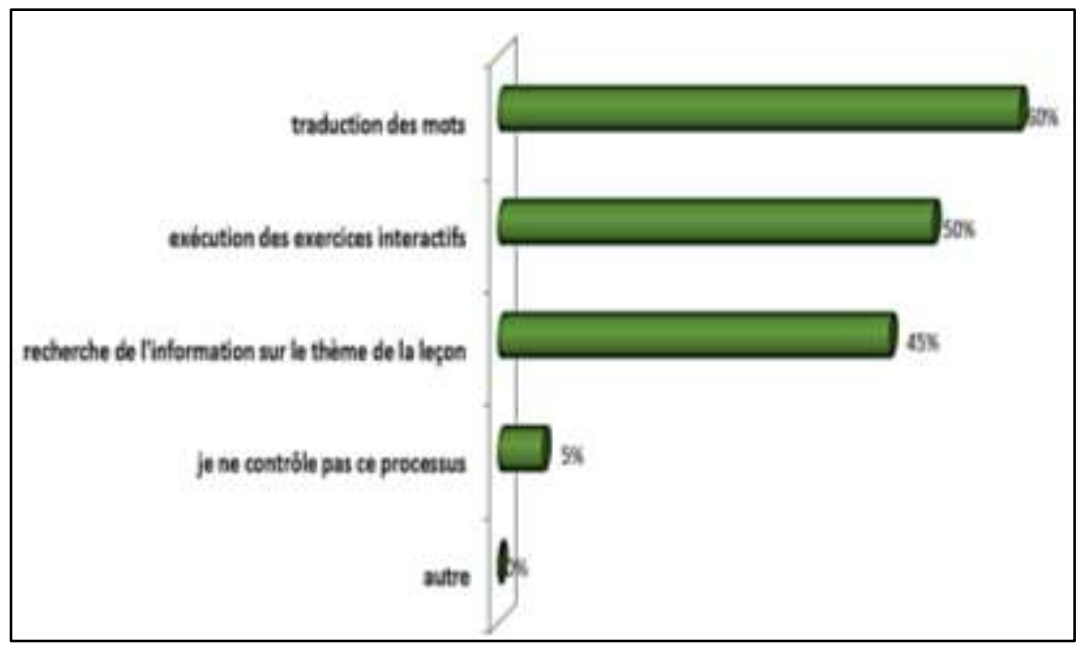

Graphique 16 : Les formes du travail pour lesquelles les professeurs autorisent les étudiants à utiliser les appareils électroniques aux cours de LV

Mais quelle est la réaction des enseignants face à l'utilisation des ON par les étudiants en cours de LV ? (Graphique 17). 43\% des enseignants en profitent et effectuent l'accompagnement pédagogique en montrant le meilleur usage éducatif des ON. Un quart d'entre eux est contre le recours aux smartphones pendant le cours et même interdit de les utiliser. Seulement $18 \%$ des professeurs s'intéressent aux applications utilisées par les étudiants. Encore moins encouragent à les utiliser (8\%). Le pourcentage de ceux qui interdisent cet usage est minime (3\%).

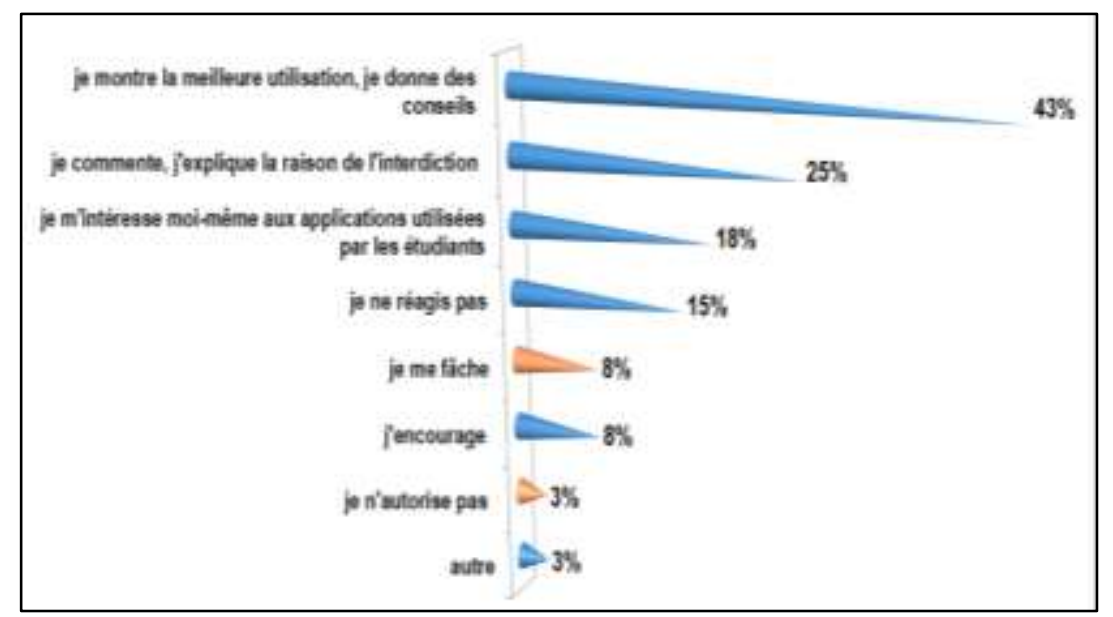

Graphique 17 : La réaction des professeurs à l'utilisation des appareils électroniques par les étudiants en classe de LV

Comment les étudiants réagissent-ils à l'interdiction d'utiliser leurs smartphones dans les salles de classe?

XLinguae, Volume 11 Issue 1XL, January 2018, ISSN 1337-8384, eISSN 2453-711X 
Un tiers continue de les utiliser secrètement, mais presque la moitié comprend cette interdiction. Il y a quand même ceux (3\%) qui éprouvent une forte réaction négative (l'agressivité, l'irritation).

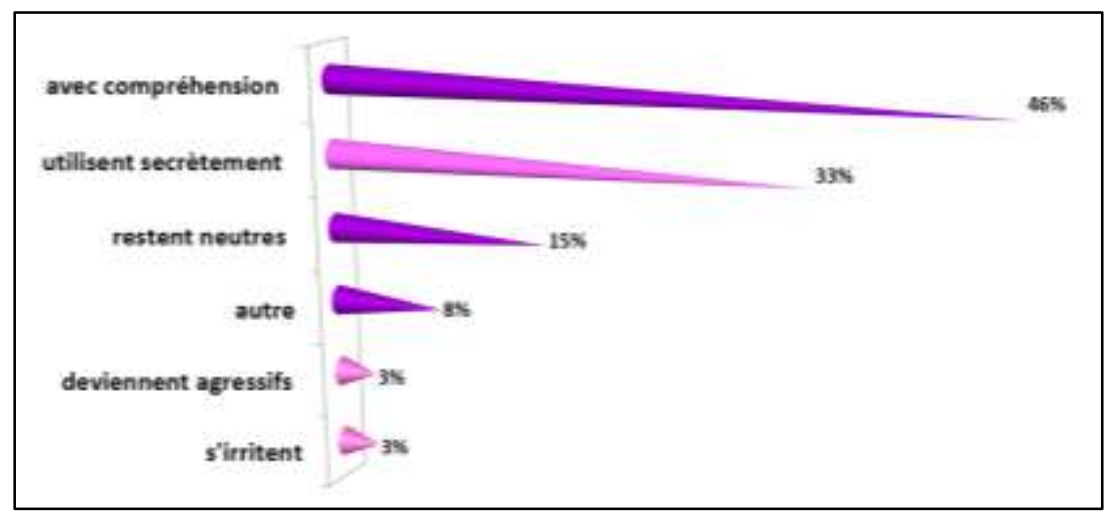

\section{Graphique 18 : La réaction des étudiants à l'interdiction de l'utilisation des appareils électroniques en classe}

Après avoir interrogé les étudiants sur le développement de leur capacités cognitives aux cours de LV nous avons décidé d'étudier les opinions des enseignants. Leurs réponses sont présentées dans le Graphique 19. Les tendances négatives sont présentées par la couleur orange et positives en vert. Presque 60\% des professeurs sont persuadés que les étudiants perdent l'autonomie de la pensée. C'est d'après cette catégorie que s'informer devient plus importante que réfléchir. Les professeurs remarquent que la rapidité de la recherche des mots avec les OU ne garantit pas leur mémorisation, les étudiants les oublient aussi vite.

Un quart des enseignants pensent que les étudiants se sentent impuissants sans gadgets. Les statistiques distinguent deux groupes d'enseignants qui constatent que les étudiants peuvent exécuter plusieurs taches simultanément, mais pour le premier groupe - $18 \%$ des interrogés cela diminue l'efficacité de leur activité cognitive à cause de la diminution de la concentration et de l'attention. Pour le deuxième groupe $(15 \%)$ l'usage des $\mathrm{ON}$ n'entraine pas une perte dans la qualité du travail.

Seulement $10 \%$ des professeurs sont persuadés que l'usage de appareils électroniques améliore l'efficacité d'apprentissage des LV, encore moins (3\%) considèrent que c'est un facteur de motivation pour les étudiants. 


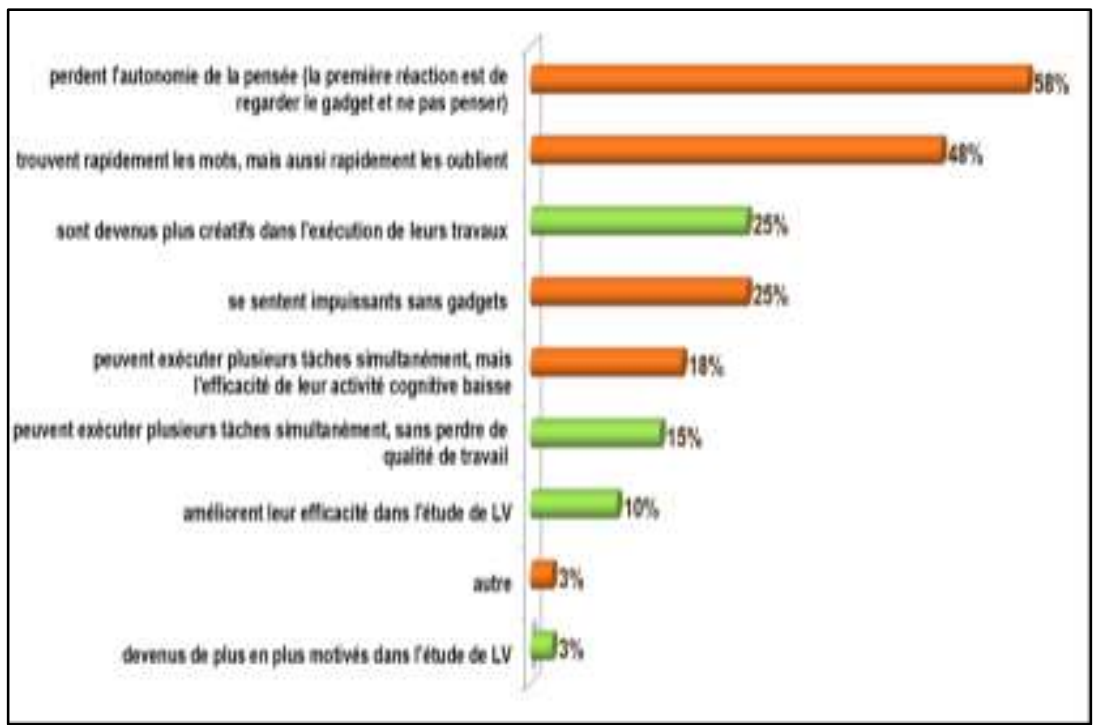

Graphique 19: Les réponses des enseignants à la suggestion « $D$ 'après mes observations, en raison de la référence constante aux $\mathrm{AN}$, les étudiants: ... »

Quelles sont les stratégies individuelles d'utilisation des TICE des enseignants des LV ? (Graphique 20). Il est à noter que 100\% des professeurs comprennent l'importance de les avoir et $70 \%$ les ont déjà. Pour presque un tiers c'est une élaboration des étapes des cours avec usage des outils numériques. 15\% expliquent les points forts et faibles d'usage des TICE lors des cours. La quantité de ceux qui encouragent à utiliser les ON pour l'apprentissage de LV reste très faible $(5 \%)$.

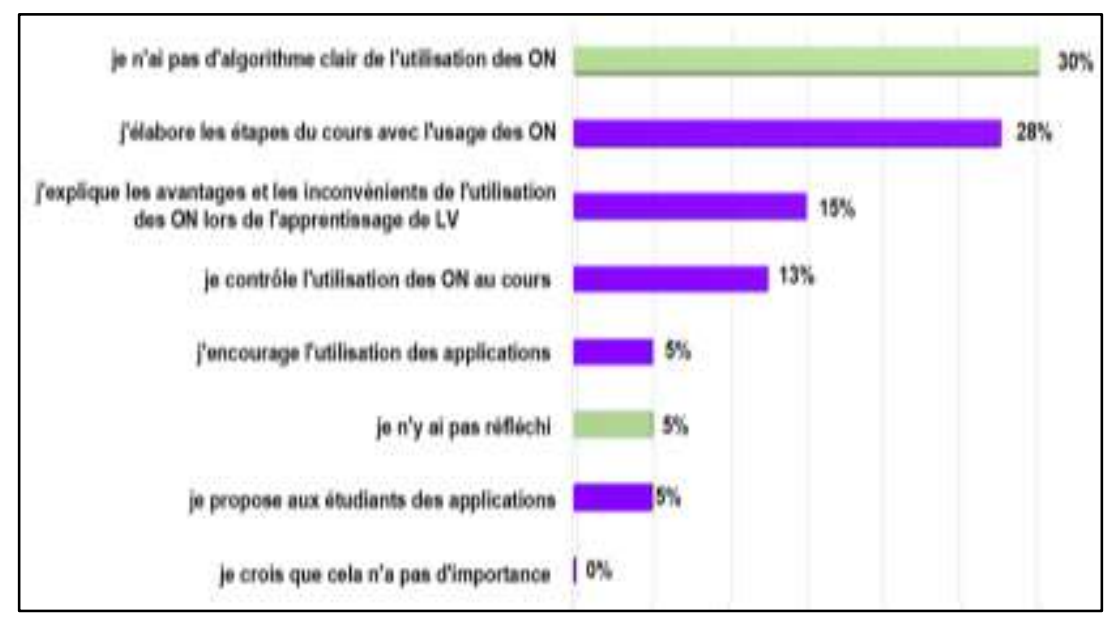

Graphique 20: Les réponses des enseignants à la confirmation « J'ai un algorithme clair de l'utilisation des outils numériques $(\mathrm{ON})$ lors de l'enseignement des LV:... » 
Dans la structure des cours, les enseignants prêtent attention à l'explication des avantages et des inconvénients de l'utilisation des $\mathrm{ON}$ de la manière suivante (Tableau 4) :

\begin{tabular}{|l|l|}
\hline Réponses des professeurs & $\%$ \\
\hline je dédie à ce sujet un cours spécial & $0 \%$ \\
\hline je donne des explications dans une situation concrète & $83 \%$ \\
\hline je ne le fais pas & $13 \%$ \\
\hline une autre possibilité & $3 \%$ \\
\hline
\end{tabular}

\section{Tableau 4 : Formes d'explication des avantages et des inconvénients des $\mathrm{ON}$ pour apprentissage des $L V$ dans la structure des cours de $L V$}

Ci-dessous nous proposons des fragments des suggestions des professeurs quant 'à l'avenir de l'étude des LV.

"Il n'y a de nouveau que ce qui est oublié "

"Parfois il est difficile d'imaginer ce qui nous attend à l'avenir »

"A notre époque, il est impossible d'imaginer la vie sans appareil électronique, mais, à mon avis, le fait de passer entièrement à leur utilisation conduit à la dégradation de la personne humaine, il y a maintenant une tendance : les élèves ne peuvent pas exprimer leur pensée de manière claire et compréhensible et surtout en langue correcte et c'est, à mon avis, à cause de l'utilisation permanente des différents appareils électroniques. Dans l'idéal, il serait bien d'avoir un juste milieu ».

"Il faut profiter des ON lors de l'apprentissage des LV, il y a plus d'avantages que d'inconvénients »

"Je pense que l'avenir de l'apprentissage des LV ne changera pas fortement - pour apprendre la LV il faut faire beaucoup d'efforts. Les ON peuvent rendre ce processus plus amusant et intéressant, mais je ne pense pas que cela améliore la qualité. On a toujours besoin de l'enseignant, qui va contrôler et corriger les erreurs pour parler correctement $"$.

\section{IV.2.3. Professeurs. Discussion.}

Pour mieux comprendre les résultats de la recherche effectuée, il faut faire attention au fait que parmi les professeurs interrogés la plus grande partie était représentée par les personnes expérimentées, qui pouvaient en s'appuyant sur leur expérience précédente comparer les méthodes, les styles d'apprentissage et le comportement des apprenants équipés ou non des gadgets à l'époque actuelle et auparavant.

En général on peut constater que les professeurs de LV sont assez critiques face aux changements "numériques» en comprenant leurs avantages et inconvénients. Ils comprennent qu'il faut s'adapter à la nouvelle réalité - intégration massive des TIC dans le processus d'enseignement-apprentissage des LV mais parallèlement $1 / 3$ des enseignants n'a pas d'algorithme individuel clair d'utilisation des TIC (85\% explications « situationnelles »).

En prenant en considération une très grande charge horaire des enseignants (jusqu'à $900 \mathrm{~h}$ par an) et que l'usage professionnel des TICE demande beaucoup de temps (pour $45 \%$ cela limite leur application) seulement un petit pourcentage (5\%) est capable de proposer des applications à utiliser pour apprendre les LV.

L'absence de bon équipement des salles d'études dans certains établissements et de bonne connexion Internet limite la motivation des enseignants à se perfectionner dans cette voie. 
En même temps les professeurs ne profitent pas des connaissances des étudiants dans ce domaine et de leurs compétences informatiques avancées $(18 \%$ s'intéressent aux applications utilisées par les étudiants).

La plupart des professeurs en travaillant avec le numérique restent «fidèles » aux supports authentiques sans beaucoup varier leurs activités.

Nous trouvons intéressantes les observations des professeurs sur l'augmentation de la dépendance des apprenants aux appareils électroniques et du changement de leur algorithme d'activité mentale. D'après eux la référence constante des étudiants aux $\mathrm{ON}$ a deux fois plus de conséquences négatives que positives.

Dans ce système de coordonnées, proposée par le contexte concret les professeurs constatent que l'usage des TIC ne favorise pas le développement des compétences cognitives des apprenants. Ce qui est évidant dans l'absence d'une vision à plus long terme des possibilités des TICE, de leur propre expérience dans ce domaine et de l'élaboration de sa propre stratégie.

En même temps les observations des professeurs nous aident à envisager les pistes de recherche dans le domaine des ON par rapport à l'enseignement-apprentissage des LV et attire notre attention sur la nécessité :

D'être plus attentif à la formation du sentiment de confiance en soi des apprenants, de ne pas avoir peur de réfléchir sans gadget

De s'informer davantage sur les applications pour la mémorisation du lexique afin de compenser le rapide oubli de l'information

De résister à la primitivité des réflexions en utilisant les riches possibilités de la discipline de LV

Prendre l'habitude de s'intéresser et de motiver les étudiants à s'informer sur de nouvelles applications pour l'apprentissage des LV et en discuter en classe

\section{Conclusions.}

1. On constate la différente perception de l'utilisation des TIC par les professeurs et les apprenants. Les réponses des étudiants correspondent plutôt à l'option « réponse correcte » qu'à la situation réelle (d'après leurs propres commentaires suite au sondage). Les deux groupes voient différemment la dépendance aux $\mathrm{ON}$.

2. L'usage des TIC n'est pas une option prioritaire dans la pratique de l'enseignement des LV par les professeurs interrogés ainsi que dans la recherche des auteurs russophones.

3. L'algorithme d'adaptation à de nouvelles pratiques dans l'enseignementapprentissage des LV est formé plutôt spontanément ce qui reflète, à notre avis, l'étape d'accumulation d'expérience dans ce domaine.

4. Dans la pratique quotidienne des enseignants il manque la vision synthétique/globale des changements dans l'apprentissage avec le numérique au niveau éducatif et cognitif.

5. Les travaux/la recherche sur le potentiel de la discipline des LV dans le développement des compétences cognitives des apprenants avec les TIC vont réduire le pessimisme des professeurs des LV au moment actuel.

Cette publication a été préparée avec le soutien du «programme RUDN 5-100 / / This publication was prepared with the support of the « RUDN University Program 5$100 »$.

XLinguae, Volume 11 Issue 1XL, January 2018, ISSN 1337-8384, eISSN 2453-711X 


\section{Bibliographic references}

BORISOVA, O. V. - VASBIEVA, D. G. - MALYKH, N. I. - VASNEV, S. A., BIROVA, J. 2016. Problem of using innovative teaching methods for distance learning students. International Electronic Journal of Mathematics Education, 11(5), 1175-1184. ISSN 2468-4945.

DEMAIZIERE, F - GROSBOIS, M. 2014. Numerique et enseignement-apprentissage des langues en Lansad - Quand, comment, pourquoi ? Alsic [En ligne], Vol. 17 | 2014, mis en ligne le 25 avril 2014, Available online: http://journals.openedition.org/alsic/2691; DOI : 10.4000/alsic.2691.

CIEKANSKI, M. 2014 «Accompagner l'apprentissage des langues à l'heure du numerique - Evolution des problematiques et diversite des pratiques ", Alsic [En ligne], Vol. 17 | 2014, mis en ligne le 17 novembre 2014, Available online: http://journals.openedition.org/alsic/2762; DOI: 10.4000/alsic.2762.

GUICHON, N. 2012. L'apprentissage des langues mediatise par les technologies (ALMT) - Etude d'un domaine de recherche emergent a travers les publications de la revue Alsic [Full text] Published in Alsic, Vol. 15, n. 3

MEZHDUNARODNAYA KONFERENCIYA «YAzyk i dejstvitel'nost'. Nauchnye chteniya na kafedre romanskih yazykov im. V.G. Gaka ». 2016 " Moskovskij pedagogicheskij gosudarstvennyj universitet» (Moskva, yanvar' 2016) (programma).

GROSBOIS, M. 2015 S'approprier le numerique : reussite ou echec ? Pour qui, pourquoi, comment? Recherche et pratiques pedagogiques en langues de specialite [En ligne], Vol. XXXIV N ${ }^{\circ} 2 \mid 2015$, mis en ligne le 29 juin 2015, Available online: http://journals.openedition.org/apliut/5168; DOI: 10.4000/apliut.5168.

INOSTRANNYE YAZYKI V NAUKE I OBRAZOVANII: PROBLEMY I PESPECTIVY. I mezhdunarodnaya nauchno-prakticheskaya konferenciya. 2015. Nacional'nyj issledovatel'skij universitet «Vysshaya shkola ehkonomiki». (Moskva, dekabr 2015) (programma).

INFORMACIONNO-KOMMUNICACIONNYE TEKHNOLOGII V LINGVISTIKE, LINGVODIDACTIKE I MEZJKULTURNOJ KOMMUNIKACII. 2014.Vypusk 6. Sbornik statej //M-vo obrazovaniya i nauki RF [i dr.] pod redakc. A.L. Nazarenko. M.: Centr Distanc. Obraz: Fakul'tet inostr.yaz. i regionovedeniya MGU imeni M.V. Lomonosova; Izd-vo «Universitetskaya kniga». - Moskva, p. 498 Available online: http://elibrary.ru/item.asp?id=23234421 ISBN: 978-5-91304-409-9.

KARSANTI, T. - KOZARENKO, O. M. 2016. Novaya model' informacionnyh kompetencij studentov i vozmozhnosti ee primeneniya $\mathrm{v}$ prepodavanii/izuchenii inostrannyh yazykov. In Lingvometodicheskie i psihologo-pedagogicheskie aspekty prepodavaniya professional'no orientirovannogo inostrannogo yazyka $\mathrm{v}$ vuze : monografiya koll. avtorov pod red. professora M.V. Mel'nichuk.-M.: Nauchnye tekhnologii pp. 204-241, Moskva. ISBN 978-5-4443-0090-9.

KASHCHUK, S. M. 2014a. Le fonctionnement de la langue française sur la toile. Inostrannye yazyki v shkole, (№ 5):56-59. ISSN0 130-6073.

KASHCHUK, S. M. 2014b. Tekhnologicheskie innovacii i innovacionnye pedagogicheskie tekhnologii $\mathrm{v}$ obuchenii inostrannym yazykam (na primere francuzskogo yazyka). Prepodavatel' XXI vek, (1):29-34. ISSN: 2073-9613.

KOZARENKO, O.M. 2016. Informacionno-kommunikacionnye tekhnologii v prepodavanii inostrannyh yazykov: chto i kak my izuchaem. V sbornike: Informacionno-kommunikacionnye tekhnologii $\mathrm{v}$ lingvistike, lingvodidaktike $\mathrm{i}$ mezhkul'turnoj kommunikacii sbornik statej. Pod redakciej A.L. Nazarenko. Moskva, 02-03 iyunya 2016 g.Organizatory: MGU imeni M.V. Lomonosova pp. 288-296. ISBN: 978-5-91304-409-9 Izdatel'stvo: ID Universitetskaya kniga (Moskva) BBK: 81ya43 UDK: 81'1(082.1).

MAGIYA INNO: novoe $\mathrm{v}$ issledovanii yazyka i metodike ego prepodavaniya mater. Vtoroj nauchn. - prakt. konf. otv. red.: D.A. Kryachkov. - Moskva, 2015. S. I tom. S. 674 http://elibrary.ru/item.asp?id=25247314 - II tom. p.676 Available online: 
http://www.inno-conf.mgimo.ru/i/Tom-2_inno-magic-2015.pdf ISBN 978-5-92281397-6 (т. 1) ISBN 978-5-9228-1399-0.

NAZARENKO, A. L. 2014. Informacionno-kommunikacionnye tekhnologii v lingvodidaktike:distancionnoe obuchenie. Mezhdunarodnyj zhurnal ehksperimental'nogo obrazovaniya, 1(11). pp.91-92. ISSN 1996-3947.

SHADRIKOV, V. D., SHEMET I. S. 2009 Informacionnye tekhnologii v obrazovanii: plyusy i minusy // Vysshee obrazovanie v Rossii. N. 11. Available online: http://cyberleninka.ru/article/n/informatsionnye-tehnologii-v-obrazovaniiplyusy-i-minusy ISSN 1996-3947.

TITOVA, S. V. 2017a. Cifrovye tekhnologii v obuchenii inostrannym yazykam: teoriya i praktika. EHditus Moskva. ISBN: 978-5-00058-548-1.

TITOVA, S. V. 2014a. Informacionno-kommunikacionnye tekhnologii v gumanitarnom obrazovanii. Ikar Moskva. ISBN: 978-5-7974-0371-5.

TITOVA, S. V. - FILATOVA, A. V. 2014b. Tekhnologii Veb $2.0 \mathrm{v}$ prepodavanii inostrannyh yazykov. Ikar Moskva. ISBN: 978-5-7974-0372-2.

VOJSKUNSKIJ, A. E. 2005. Psihologicheskie issledovaniya deyatel'nosti cheloveka v Internete. Informacionnoe obshchestvo, (1), pp.36-41. ISSN 2079-6617.

Words: 6230

Characters: 41488 (23,05 standard pages)

Assoc. Prof. Dr. Olga Kozarenko

Department of theory and practice of foreign languages

Russian Peoples Friendship University, Institute of Foreign languages

Mikluho-Maklaya, 7 Moscou 117198

Russian Federation

kozarenko_om@rudn.university

XLinguae, Volume 11 Issue 1XL, January 2018, ISSN 1337-8384, eISSN 2453-711X 